\title{
LOGARITHMIC INTERPRETATION OF THE MAIN COMPONENT IN TORIC HILBERT SCHEMES
}

\author{
MARTIN OLSSON
}

\section{Statement of theOrem}

We work over a base field $k$, except in the last section.

1.1. Fix a surjective morphism of fine monoids $\pi: P \rightarrow Q$, and let $j: A_{Q} \hookrightarrow A_{P}$ be the corresponding closed immersion, where $A_{P}:=\operatorname{Spec}(k[P])$ and $A_{Q}:=\operatorname{Spec}(k[Q])$. Assume further that the associated groups $P^{g p}$ and $Q^{g p}$ are torsion free, and that $Q$ is sharp. Let $T_{P}$ (resp. $T_{Q}$ ) denote the torus associated to the group $P^{g p}$ (resp. $Q^{g p}$ ) so that $T_{P}$ acts on $A_{P}$ and $T_{Q}$ acts on $A_{Q}$. The map $\pi$ induces an inclusion of tori $\pi_{T}: T_{Q} \rightarrow T_{P}$, and the closed immersion $j$ is compatible with the action of $T_{Q}$. Define a function $h: Q^{g p} \rightarrow \mathbb{N}$ by

$$
h(q):= \begin{cases}1 & \text { if } q \in Q, \\ 0 & \text { otherwise. }\end{cases}
$$

We can then consider the multigraded Hilbert functor of Haiman and Sturmfels [H-S]

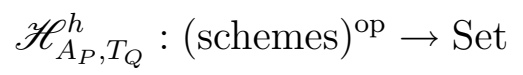

sending a scheme $S$ to the set of $T_{Q}$-invariant closed immersions $Z \hookrightarrow A_{P, S}$ over $S$ such that if $g: Z \rightarrow S$ is the structure morphism then for every $q \in Q^{g p}$ the $q$-eigenspace $\left(g_{*} \mathcal{O}_{Z}\right)_{q}$ of $g_{*} \mathcal{O}_{Z}$ is a finitely presented projective $\mathcal{O}_{S}$-module of $\operatorname{rank} h(q)$. By [H-S, 1.1] the functor $\mathscr{H}_{A_{P}, T_{Q}}^{h}$ is representable by a quasi-projective scheme. To ease notation in what follows we write simply $\mathscr{H}$ for $\mathscr{H}_{A_{P}, T_{Q}}^{h}$ (since the above data will be fixed throughout the paper).

A (scheme-valued) point $u \in T_{P}(S)$ defines a map $u^{*}: \mathscr{H}(S) \rightarrow \mathscr{H}(S)$ by sending $i: Z \hookrightarrow A_{P, S}$ to the composite

$$
Z \stackrel{i}{\longrightarrow} A_{P, S} \stackrel{u}{\longrightarrow} A_{P, S}
$$

Note that since $Z$ is $T_{Q}$-invariant this map $u^{*}$ depends only on the image of $u$ in $T_{P} / T_{Q}=T_{K}$, where $K:=\operatorname{Ker}\left(P^{g p} \rightarrow Q^{g p}\right)$. We therefore obtain an action of $T_{K}$ on $\mathscr{H}$.

There is a distinguished point $p_{0} \in \mathscr{H}(\operatorname{Spec}(k))$ given by $j: A_{Q} \hookrightarrow A_{P}$. This defines a $T_{K}$-equivariant morphism (where $T_{K}$ acts on itself by translation)

$$
z: T_{K} \rightarrow \mathscr{H}, \quad u \mapsto u * p_{0} .
$$

By $[\mathrm{C}, 3.4]$ the map $z$ is an open immersion. We define the main component of $\mathscr{H}$ to be the scheme-theoretic closure of the image $z\left(T_{K}\right)$. Let $\mathscr{S}_{P \rightarrow Q}$ denote the normalization of the main component of $\mathscr{H}$.

The problem we propose a solution to in this note is the following: 
Problem 1.2. Define a reasonable (i.e. not the functor of points) functor which is represented by $\mathscr{S}_{P \rightarrow Q}$.

1.3. The scheme $\mathscr{S}_{P \rightarrow Q}$ with its action of $T_{K}$ is a normal toric variety and therefore has a natural fs $\log$ structure $M_{\mathscr{S}_{P \rightarrow Q}}$ such that $\left(\mathscr{S}_{P \rightarrow Q}, M_{\mathscr{S}_{P \rightarrow Q}}\right)$ is $\log$ smooth over $k$. We will show that the fs $\log$ scheme $\left(\mathscr{S}_{P \rightarrow Q}, M_{\mathscr{S}_{P \rightarrow Q}}\right)$ represents a logarithmic version of the multigraded Hilbert scheme.

1.4. Let $M_{A_{P}}$ (resp. $M_{A_{Q}}$ ) denote the fine $\log$ structure on $A_{P}$ (resp. $A_{Q}$ ) induced by the natural map $P \rightarrow k[P]$ (resp. $Q \rightarrow k[Q]$ ) so that the inclusion $A_{Q} \hookrightarrow A_{P}$ extends to a closed immersion of fine log schemes

$$
\left(A_{Q}, M_{A_{Q}}\right) \hookrightarrow\left(A_{P}, M_{A_{P}}\right) .
$$

Note that the action of $T_{Q}$ (resp. $T_{P}$ ) extends naturally to an action on $\left(A_{Q}, M_{A_{Q}}\right)$ (resp. $\left(A_{P}, M_{A_{P}}\right)$ ) and the closed immersion 1.4.1 is $T_{Q}$-equivariant.

1.5. Define a functor (the logarithmic toric Hilbert functor)

$$
\mathscr{H}^{\log }:(\text { fs } \log \text { schemes } / k)^{\text {op }} \rightarrow \text { Set }
$$

by associating to any fs-log scheme $\left(S, M_{S}\right)$ over $k$ the set of commutative diagrams

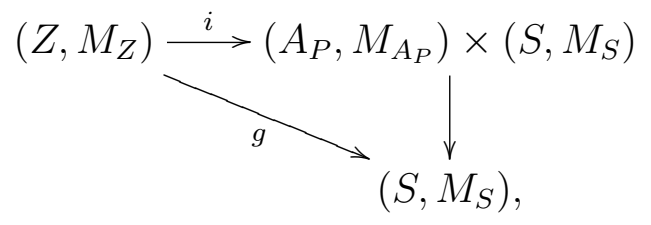

such that the following hold:

(i) $i$ is a $T_{Q}$-invariant closed immersion of fine log schemes;

(ii) $g$ is $\log$ smooth and integral (this implies in particular that the underlying morphism $Z \rightarrow S$ is flat);

(iii) For every $q \in Q^{g p}$ the $q$-eigenspace $\left(g_{*} \mathcal{O}_{Z}\right)_{q}$ is a finitely presented projective $\mathcal{O}_{S^{-}}$ module of rank $h(q)$.

(iv) The map

$$
P \rightarrow M_{\left(Z, M_{Z}\right) /\left(S, M_{S}\right)}:=\operatorname{Coker}\left(g^{*} M_{S} \rightarrow M_{Z}\right)
$$

induced by $i$ factors through $Q$.

Remark 1.6. In the case when the log structure on $S$ is trivial, condition (iii) enables one to recover the $\log$ structure $M_{Z}$ on $Z$ from the underlying scheme. Indeed the monoid $Q$ is obtained from the condition that $q \in Q^{g p}$ is in $Q$ if and only if the eigenspace $\left(g_{*} \mathcal{O}_{Z}\right)_{q}$ is nonzero. Since each module $\left(g_{*} \mathcal{O}_{Z}\right)_{q}$ is locally free of rank 1 , there is a $T_{Q}$-invariant open subscheme $Z^{*} \subset Z$ obtained by inverting local generators of the modules $\left(g_{*} \mathcal{O}_{Z}\right)_{q}$ for $q \neq 0$. Condition (ii) implies that for every geometric point $\bar{s} \rightarrow S$ the fiber $Z_{\bar{s}}^{*} \subset Z_{\bar{s}}$ is dense, and in fact equal to the maximal open $T_{Q}$-invariant subset in the toric variety $Z_{\bar{s}}$. From this it also follows that if $h: Z^{*} \rightarrow S$ is the projection, then $\left(h_{*} \mathcal{O}_{Z^{*}}\right)_{q}$ is a projective module of rank 1 for all $q \in Q^{g p}$. Let $q \in Q$ be an element and let $p_{1}, p_{2} \in P$ be two liftings of $q$ to $P$. Let $\beta_{1}, \beta_{2} \in \mathcal{O}_{Z}$ be the images of $p_{1}$ and $p_{2}$. Then $\beta_{1}$ and $\beta_{2}$ map to units in $h_{*} \mathcal{O}_{Z^{*}}$ and $\beta_{1} \cdot \beta_{2}^{-1}$ is $T_{Q^{-}}$invariant. Since the map $g_{*} \mathcal{O}_{Z} \rightarrow h_{*} \mathcal{O}_{Z^{*}}$ is injective, it follows that there exists a unique unit $u \in \mathcal{O}_{S}^{*}$ such that $\beta_{1}=u \beta_{2}$. As above let $K$ denote the kernel of $P^{g p} \rightarrow Q^{g p}$. 
The preceding discussion implies that the map $P \rightarrow \mathcal{O}_{Z}$ induces a map $P_{K} \rightarrow \mathcal{O}_{Z}$ from the localization $P_{K}$ of $P$, and the associated $\log$ structure is equal to $M_{Z}$.

It follows from this that the composite functor

$$
\text { (schemes) })^{\text {op }} \stackrel{S \mapsto\left(S, \mathcal{O}_{S}^{*}\right)}{\longrightarrow}(\text { fs } \log \text { schemes })^{\text {op }} \stackrel{\mathscr{H}^{\log }}{\longrightarrow} \text { Set }
$$

is representable by the open image of $z: T_{K} \rightarrow \mathscr{H}$.

A basic idea in the above definition of $\mathscr{H}^{\text {log }}$ is that by allowing nontrivial log structures $M_{S}$ on the base $S$, we also include specializations (i.e. degenerations) of points in $z\left(T_{K}\right)$, but the log deformation theory only allows deformations in the main component.

The main result of this paper, whose proof occupies the subsequent sections, is the following (see 2.9 for the morphism of functors relating $\mathscr{H}^{\log }$ and $\left(\mathcal{S}_{P \rightarrow Q}, M_{\mathcal{S}_{P \rightarrow Q}}\right)$ ):

Theorem 1.7. The functor $\mathscr{H}^{\log }$ is represented by $\left(\mathcal{S}_{P \rightarrow Q}, M_{\mathcal{S}_{P \rightarrow Q}}\right)$.

Note that the definition of $\mathscr{H}^{\text {log }}$ in 1.5 makes sense not just over a field $k$ but over $\mathbb{Z}$. In fact the only time in the above discussion where the base field is relevant is in the definition of the main component (since scheme-theoretic closure does not commute with base change), and the fact that the normalization of the main component is a toric variety. Write

$$
\mathscr{H}_{\mathbb{Z}}^{\log }:(\text { fs } \log \text { schemes }) \rightarrow \text { Set }
$$

for this functor, so that for the field $k$ above the functor $\mathscr{H}^{\log }$ is obtained by restricting $\mathscr{H}_{\mathbb{Z}}^{\log }$ to the category of fs log schemes over $k$. In the last section we explain how to generalize 1.7 to show that $\mathscr{H}_{\mathbb{Z}}^{\text {log }}$ is representable by an integral version of $\left(\mathcal{S}_{P \rightarrow Q}, M_{\mathcal{S}_{P \rightarrow Q}}\right)$.

Remark 1.8. As pointed out to us by Sturmfels, one of the very appealing aspects of the multigraded Hilbert scheme is that tangent spaces of points can be computed by a simple formula. We discuss this and the relationship with the logarithmic tangent space of the toric variety $\mathcal{S}_{P \rightarrow Q}$ in 4.5 .

1.9. (Notation). For a group $G$, we write $D(G)$ for the diagonalizable group scheme associated to $G$.

For a fine monoid $P$ and ring $R$, we denote by $R[P]$ the monoid algebra on $P$, and for $p \in P$ we write $e_{p} \in R[P]$ for the image of $p$ under the canonical map $P \rightarrow R[P]$.

1.10. (Acknowledgements). The author is grateful to Bernd Sturmfels for suggesting the problem in this paper, and Arthur Ogus for helpful conversations.

This work was partially supported by NSF grant DMS-0714086 and an Alfred P. Sloan Research Fellowship. Much of this work was done while the author visited the Institute Mittag-Leffler (Djursholm, Sweden). The author is grateful for the Institute's hospitality and excellent working conditions.

\section{LOGARITHMIC STRUCTURE ON THE FAMILY OVER $\mathcal{S}_{P \rightarrow Q}$}

2.1. The morphism $\mathcal{S}_{P \rightarrow Q} \rightarrow \mathscr{H}$ corresponds to a closed subscheme $j: Z \hookrightarrow A_{P} \times \mathcal{S}_{P \rightarrow Q}$. Let $M_{A_{P} \times \mathcal{S}_{P \rightarrow Q}}$ be the product log structure on $A_{P} \times \mathcal{S}_{P \rightarrow Q}$ (given by $p_{1}^{*} M_{A_{P}} \oplus_{\mathcal{O}^{*}} p_{2}^{*} M_{\mathcal{S}_{P \rightarrow Q}}$ with its canonical map to the structure sheaf). 
2.2. To construct the log structure on $z$, consider first the case when $k$ is algebraically closed. In this case the toric variety $\mathcal{S}_{P \rightarrow Q}$ is given by a fan in $K_{\mathbb{R}}$ (recall that $K$ is the kernel of $P^{g p} \rightarrow Q^{g p}$ ). Let us describe this fan in more detail (this description is derived from $[\mathrm{C}]$ ). Let $L \subset K$ be a submonoid such that $\operatorname{Spec}(k[L])$ is an open subset of $\mathcal{S}_{P \rightarrow Q}$. The restriction of $z$ to $\operatorname{Spec}(k[L])$ can then be described as follows.

Let $E \subset Q \oplus P^{\text {gp }}$ be the submonoid of elements $(q, p)$ such that $\pi(p)=q$.

Lemma 2.3. The restriction of $\mathcal{Z} \hookrightarrow A_{P} \times \mathcal{S}_{P \rightarrow Q}$ to $\operatorname{Spec}(k[K]) \subset \mathcal{S}_{P \rightarrow Q}$ is equal to the closed immersion

$$
\operatorname{Spec}(k[E]) \hookrightarrow \operatorname{Spec}(k[P \oplus K])
$$

induced by the morphism of monoids $\gamma: P \oplus K \rightarrow E \subset Q \oplus P^{g p} \operatorname{sending}(p, l)$ to $(\pi(p), p+l)$.

Proof. Let $R$ be a ring and let $\rho_{0}: K \rightarrow R^{*}$ be the constant morphism sending all elements to 1 . Then the point $p_{0} \in \mathscr{H}(\operatorname{Spec}(R))$ is given by the surjection

$$
R[P] \simeq R \otimes_{\rho_{0}, R[K]} R[P \oplus K] \longrightarrow R \otimes_{\rho_{0}, R[K]} R[E] \simeq R[Q] .
$$

Now let $\rho: K \rightarrow R^{*}$ be another homomorphism. We show that the $R$-valued point

$$
\left(Z_{R} \hookrightarrow A_{P} \times \operatorname{Spec}(R)\right) \in \mathscr{H}(\operatorname{Spec}(R))
$$

defined by the base change of $z$ along $\rho: k[K] \rightarrow R$ is equal to $\rho * p_{0}$.

To verify this we may replace $R$ by a finite flat extension, and hence we may assume there exists an extension $\tilde{\rho}: P \rightarrow R^{*}$ of $\rho$. This extension also induces a morphism $\rho_{E}: E \rightarrow R^{*}$ by taking the composite

$$
E \subset Q \oplus P^{g p} \stackrel{\text { pr }}{\longrightarrow} P^{g p} \stackrel{\tilde{\rho}}{\longrightarrow} R^{*} .
$$

The lemma then follows by noting that there is a commutative diagram

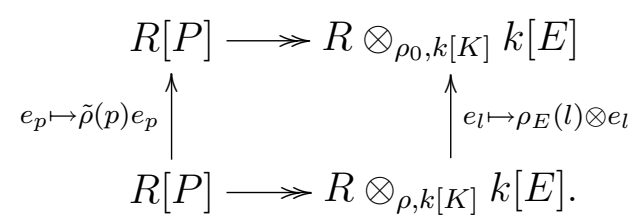

2.4. Let $R$ be the coordinate ring of $Z \times_{s_{P \rightarrow Q}} \operatorname{Spec}(k[L])$ so we have a commutative diagram of rings

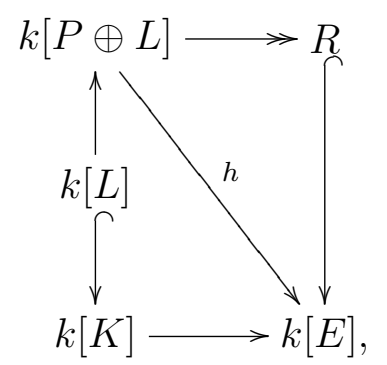

where the map $h$ is induced by the map of monoids $\gamma$. It follows that if $E_{L} \subset E$ denotes the image of $P \oplus L$, then $R$ is equal to the monoid algebra $k\left[E_{L}\right]$. The morphism of monoids $L \rightarrow E_{L}$ is integral since this is equivalent to the flatness of the map of rings $k[L] \rightarrow k\left[E_{L}\right]$ 
[Og1, 4.3.7]. Conversely, if we start with a submonoid $L \subset K$ and the resulting map $L \rightarrow E_{L}$ is integral, then

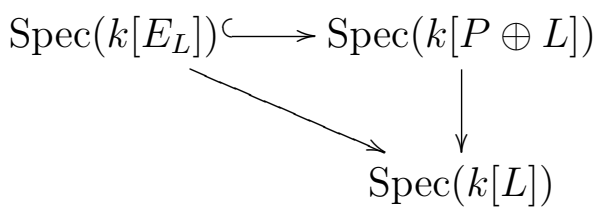

defines a $T_{K}$-equivariant morphism $\operatorname{Spec}(k[L]) \rightarrow \mathcal{S}_{P \rightarrow Q}$, and therefore $L$ lies in a cone of the fan defining $\mathcal{S}_{P \rightarrow Q}$.

Corollary 2.5. The fan $\Sigma(P \rightarrow Q)$ defining $\mathcal{S}_{P \rightarrow Q}$ is characterized by the condition that a submonoid $L \subset K$ lies in a cone of $\Sigma(P \rightarrow Q)$ if and only if $L \rightarrow E_{L}$ is integral.

2.6. Note that this characterization makes no reference to the ground field $k$. Furthermore, if $k$ is not algebraically closed and $\mathcal{S}_{P \rightarrow Q}^{\prime}$ temporarily denotes the toric variety over $k$ associated to $\Sigma(P \rightarrow Q)$, then the families 2.4.1 define maps to $\mathcal{S}_{P \rightarrow Q}$ over the torus invariant open subsets of $\mathcal{S}_{P \rightarrow Q}^{\prime}$. These maps are compatible by construction so we get a morphism $\mathcal{S}_{P \rightarrow Q}^{\prime} \rightarrow$ $\mathcal{S}_{P \rightarrow Q}$. This map of toric varieties becomes an isomorphism after base change to $\bar{k}$, and therefore is already an isomorphism over $k$. Thus even when $k$ is not algebraically closed the toric variety $\mathcal{S}_{P \rightarrow Q}$ is defined by the fan $\Sigma(P \rightarrow Q)$.

2.7. This description also enables us to define a log structure $M_{z}$ on the universal family $z \subset A_{P} \times \mathcal{S}_{P \rightarrow Q}$. Let $L \subset K$ be a submonoid such that $\operatorname{Spec}(k[L])$ defines an open subset of $\mathcal{S}_{P \rightarrow Q}$. The restriction of $z$ to $\operatorname{Spec}(k[L])$ is then equal to $\operatorname{Spec}\left(k\left[E_{L}\right]\right)$. Define $M_{z}$ to be the $\log$ structure associated to the map $E_{L} \rightarrow k\left[E_{L}\right]$. The morphism $\operatorname{Spec}\left(E_{L} \rightarrow k\left[E_{L}\right]\right) \rightarrow$ $\operatorname{Spec}(L \rightarrow k[L])$ is $\log$ smooth since the map $K=L^{g p} \rightarrow E_{L}^{g p}=E^{g p}$ is injective with cokernel equal to $Q^{g p}$ (which is torsion free by assumption). The following lemma implies that this construction is compatible with inclusions $L \hookrightarrow L^{\prime}$ of submonoids of $K$. It therefore globalizes and we get a $\log$ structure $M_{z}$ on $z$.

Lemma 2.8. Let $L \subset L^{\prime} \subset K$ be submonoids whose associated groups are equal to $K$. Then the diagram

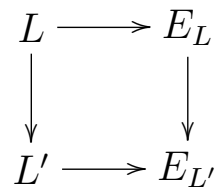

is cocartesian.

Proof. Let $N$ denote the pushout $L^{\prime} \oplus_{L} E_{L}$ so we have a map $N \rightarrow E_{L^{\prime}}$ which we want to show is an isomorphism. The surjectivity is clear by the definition of $E_{L^{\prime}}$. For the injectivity, note that the functor of taking the group associated to a monoid commutes with pushouts, and therefore $N^{g p} \simeq K \oplus_{K} E_{L}^{g p} \simeq E_{L}^{g p}$. This implies that the map $E_{L}^{g p} \rightarrow E_{L^{\prime}}^{g p}$ is injective (since both are subgroups of $Q^{g p} \oplus P^{g p}$ ), and therefore $N \rightarrow E_{L^{\prime}}$ is also injective.

2.9. Let

$$
\mathscr{H}_{S}:(\text { fs } \log \text { schemes })^{\text {op }} \rightarrow \text { Set }
$$


denote the functor of points of the log scheme $\left(\mathcal{S}_{P \rightarrow Q}, M_{\mathcal{S}_{P \rightarrow Q}}\right)$. The diagram

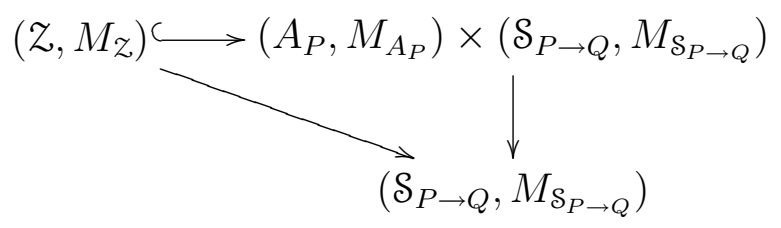

defines a $\left(\mathcal{S}_{P \rightarrow Q}, M_{\mathcal{S}_{P \rightarrow Q}}\right)$-valued point of $\mathscr{H}^{\log }$ and therefore a morphism of functors

$$
F: \mathscr{H}_{S} \rightarrow \mathscr{H}^{\log } \text {. }
$$

We show in the following sections that this morphism of functors is an isomorphism.

\section{Calculation of the log differentials}

Let $k$ be a field, $L$ a fine sharp monoid, and let $M_{k}$ denote the log structure on $\operatorname{Spec}(k)$ defined by the map $L \rightarrow k$ sending all nonzero elements to $0\left(\operatorname{so} M_{k}=\mathcal{O}_{\operatorname{Spec}(k)}^{*} \oplus L\right)$.

Recall that a log scheme $\left(T, M_{T}\right)$ is called hollow if for any local section $m \in M_{T}$ the image of $m$ in $\mathcal{O}_{T}$ is either a unit or zero. This implies that the sheaf $\bar{M}_{T}$ is a constant sheaf on each connected component of $T$ (and the converse holds if $T$ is reduced).

Proposition 3.1. Let $\left(T, M_{T}\right)$ be a hollow log scheme. Then any log smooth morphism $f:\left(T, M_{T}\right) \rightarrow\left(\operatorname{Spec}(k), M_{k}\right)$ is strict. In particular the underlying morphism of schemes $T \rightarrow \operatorname{Spec}(k)$ is smooth and $\Omega_{\left(T, M_{T}\right) /\left(k, M_{k}\right)}^{1}=\Omega_{T / k}^{1}$.

Proof. Fix a $\log$ smooth morphism $f:\left(T, M_{T}\right) \rightarrow\left(\operatorname{Spec}(k), M_{k}\right)$. The assertion that $f$ is strict is étale local on $T$. Furthermore, it suffices to verify the proposition after making a field extension of $k$, so we may assume that $k$ is algebraically closed. By $[\mathrm{Og} 1,3.3 .1]$ there exists étale locally on $T$ an injective map of monoids $\gamma: L \rightarrow N$ such that the order of the torsion part of $N^{g p} / L^{g p}$ is invertible in $k$ and a chart $\beta: N \rightarrow M_{T}$ such that the diagram

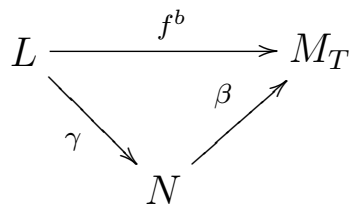

commutes, and such that the induced morphism

$$
T \rightarrow \operatorname{Spec}\left(k \otimes_{k[L]} k[N]\right)
$$

is étale. Let $t \in T(k)$ be a closed point (note that $T / k$ is locally of finite type since $f$ is $\log$ smooth). The chart $\beta$ defines a map $\beta_{t}: N \rightarrow k$. Let $F$ denote the face $\beta_{t}^{-1}\left(k^{*}\right)$, and let $N_{F}$ denote the localization of $N$ at $F$. Then the image of $t$ in $\operatorname{Spec}\left(k \otimes_{k[L]} k[N]\right)$ is contained in the open subset $\operatorname{Spec}\left(k \otimes_{k[L]} k\left[N_{F}\right]\right)$. Replacing $N$ by $N_{F}$ and shrinking correspondingly around $t$ on $T$, we may assume that $F \subset N$ is in fact equal to $N^{*}$.

Let $\left(k \otimes_{k[L]} k[N]\right)^{\wedge}$ denote the completion of $k \otimes_{k[L]} k[N]$ along the maximal ideal $\mathfrak{m}$ generated by the elements $\beta(n)-e_{n}\left(n \in N^{*}\right)$ and $e_{n}\left(n \in N-N^{*}\right)$. Since $T \rightarrow \operatorname{Spec}\left(k \otimes_{k[L]} k[N]\right)$ is étale and $T$ is hollow, we must have that the image of any $n \in N-N^{*}$ in $\left(k \otimes_{k[L]} k[N]\right)^{\wedge}$ is zero. On the other hand, consider the quotient of $k \otimes_{k[L]} k[N]$ by the ideal $\mathfrak{p}$ generated by 
$\mathfrak{m}^{2}$ and the elements $\beta(n)-e_{n}$ for $n \in N^{*}$. As a $k$-vector space the quotient $k \otimes_{k[L]} k[N] / \mathfrak{p}$ has basis given by $e_{0}$ and $e_{n}$ for a set of liftings $n \in N$ of the irreducible elements in $N / N^{*}$ which are not in the image of $L$. Since the image of $N$ in this ring is also zero we must have that the map $L \rightarrow N / N^{*}$ is an isomorphism, and so $N=L \oplus N^{*}$ and $f$ is strict.

Proposition 3.2. Let $\left(\operatorname{Spec}(k), M_{k}\right)$ be as above, and let

$$
\left(\left(Z, M_{Z}\right) \hookrightarrow\left(A_{P}, M_{A_{P}}\right) \times\left(\operatorname{Spec}(k), M_{k}\right)\right) \in \mathscr{H}^{\log }\left(\operatorname{Spec}(k), M_{k}\right)
$$

be an object.

(i) $Z$ is geometrically connected.

(ii) The rank of the locally free sheaf $\Omega_{\left(Z, M_{Z}\right) /\left(k, M_{k}\right)}^{1}$ is equal to the rank of the group $Q^{g p}$.

Proof. We may without loss of generality assume that $k$ is algebraically closed.

Let $R=\oplus_{q \in Q} R_{q}$ denote the coordinate ring of $Z$, where $R_{q}$ is the 1-dimensional vector space on which $T_{Q}$ acts through the character $q$. Statement (i) follows from the fact that $R_{0}$ is 1-dimensional.

Let $Z_{i} \subset Z$ be an irreducible component, which we view as a subscheme with the reduced structure, and let $R^{(i)}$ denote the coordinate ring of $Z_{i}$ so we have a surjection

$$
\pi_{i}: R \rightarrow R^{(i)}
$$

Since $T_{Q}$ is connected, the action of $T_{Q}$ on $Z$ preserves $Z_{i}$, and the map $\pi_{i}$ is compatible with the $T_{Q}$-actions. Let $Q^{(i)} \subset Q$ denote the subset of characters $q \in Q$ for which the $q$-eigenspace in $R^{(i)}$ is nonzero. Then $Q^{(i)} \subset Q$ is a submonoid, and $Z_{i}$ is a toric variety with action of the torus $T_{Q^{(i)}}$. Fix a $T_{Q^{(i)}}$-equivariant embedding $j_{i}: T_{Q^{(i)}} \hookrightarrow Z_{i}$. Then the image of any element of $P$ under the composite

$$
P \rightarrow \Gamma\left(Z, M_{Z}\right) \rightarrow \Gamma\left(Z_{i}, \mathcal{O}_{Z_{i}}\right) \rightarrow k\left[Q^{(i), g p}\right]
$$

is either a unit or zero, since the image of any $p \in P$ in $k\left[Q^{(i), g p}\right]$ is contained in an eigenspace for the action of $T_{Q}$. Therefore the restriction of $M_{Z}$ to $T_{Q^{(i)}} \subset Z_{i}$ is hollow. By 3.1 it follows that the rank of $\Omega_{\left(Z, M_{Z}\right) /\left(k, M_{k}\right)}^{1}$ is equal to the rank of the group $Q^{(i), g p}$. It therefore suffices to show that for some $i$ the rank of $Q^{(i), g p}$ is equal to the rank of $Q^{g p}$.

Let $J \subset R$ be the nil-radical. The nonzero eigenspaces for the $T_{Q}$-action on $R / J$ are given by the union $\cup_{i} Q^{(i)} \subset Q$. The $R / J$-module $\operatorname{gr}_{J}(R)^{+}:=\oplus_{n \geq 1} J^{n} / J^{n+1}$ is finitely generated, so there exists elements $q_{1}, \ldots, q_{r} \in Q$ such that every nonzero eigenspace occurring in $\operatorname{gr}_{J}(R)^{+}$ is of the form $q^{(i)}+q_{j}$ for some $q^{(i)} \in Q^{(i)}$ and some $q_{j}$. Since for every $q \in Q$ the eigenspace $R_{q}$ is nonzero, it follows that the union of the $Q^{(i)}$ and the finitely many translates $Q^{(i)}+q_{j}$ is equal to all of $Q$. Therefore some $Q^{(i), g p}$ must have rank equal to the rank of $Q^{g p}$.

Example 3.3. Let $P=\mathbb{N}^{2}$ with generators $e_{1}$ and $e_{2}, Q=\mathbb{N}$, and let $\pi: P \rightarrow Q$ be the map sending $e_{1}$ to 2 and $e_{2}$ to 1 . The map on tori $T_{Q} \rightarrow T_{P}$ is then the map $\mathbb{G}_{m} \rightarrow \mathbb{G}_{m}^{2}$ sending $u$ to $\left(u^{2}, u\right)$. The kernel $K$ of $P^{g p} \rightarrow Q^{g p}$ is generated by the element $(-1,2)$. Let $L \subset K$ be the submonoid generated by $(-1,2)$, and let $E_{L} \subset \mathbb{N} \oplus \mathbb{Z}^{2}$ be as in 2.4 . The map $L \rightarrow E_{L}$ is automatically integral since $L$ is generated by a single element. The monoid $E_{L} \subset \mathbb{N} \oplus \mathbb{Z}^{2}$ is the submonoid generated by the elements $(2,1,0),(1,0,1)$, and $(0,-1,2)$. From this it 
follows that $\operatorname{Spec}\left(k\left[E_{L}\right]\right) \rightarrow \operatorname{Spec}(k[L])$ is isomorphic to the family

$$
\operatorname{Spec}\left(k[t, x, y] /\left(y^{2}=t x\right)\right) \rightarrow \operatorname{Spec}(k[t]) .
$$

Note that the fiber over $t=0$ with the reduced structure is isomorphic to $k[x]$, and the corresponding submonoid of $Q=\mathbb{N}$ is the submonoid generated by 2 . Thus in general the $Q^{(i), g p} \subset Q^{g p}$ occurring in the proof of 3.2 may be strictly smaller than $Q^{g p}$ though they have the same rank.

Corollary 3.4. For any $f s$ log scheme $\left(S, M_{S}\right)$ and object 1.5.1 of $\mathscr{H}^{\log }\left(S, M_{S}\right)$, the rank of the locally free sheaf $\Omega_{\left(Z, M_{Z}\right) /\left(S, M_{S}\right)}^{1}$ is equal to the rank of $Q^{g p}$.

Proof. The rank of $\Omega_{\left(Z, M_{Z}\right) /\left(S, M_{S}\right)}^{1}$ can be calculated fiber-by-fiber so it suffices to consider the case when $S$ is the spectrum of a field.

3.5. For an fs-log scheme and object 1.5.1 of $\mathscr{H}^{\log }\left(S, M_{S}\right)$, let $\mathbb{L}_{i}$ denote the logarithmic cotangent complex (in the sense of [Ol1]) of the morphism $i$. The distinguished triangle

$$
i^{*} \Omega_{\left(A_{P}, M_{A_{P}}\right) \times\left(S, M_{S}\right) /\left(S, M_{S}\right)}^{1} \rightarrow \Omega_{\left(Z, M_{Z}\right) /\left(S, M_{S}\right)}^{1} \rightarrow \mathbb{L}_{i}
$$

shows that $\mathbb{L}_{i}$ is a complex concentrated in degree -1 and that $\mathscr{H}^{-1}\left(\mathbb{L}_{i}\right)$ is a locally free sheaf of rank equal to $\operatorname{rk}\left(P^{g p}\right)-\operatorname{rk}\left(Q^{g p}\right)=\operatorname{rk}(K)$.

Proposition 3.6. For any $f s$ log scheme $\left(S, M_{S}\right)$ and object 1.5.1 of $\mathscr{H}^{\log }\left(S, M_{S}\right)$, the map

$$
P^{g p} \otimes \mathcal{O}_{Z} \simeq i^{*} \Omega_{\left(A_{P}, M_{A_{P}} \times\left(S, M_{S}\right) /\left(S, M_{S}\right)\right.}^{1} \rightarrow \Omega_{\left(Z, M_{Z}\right) /\left(S, M_{S}\right)}^{1}
$$

induces an isomorphism

$$
Q^{g p} \otimes \mathcal{O}_{Z} \simeq \Omega_{\left(Z, M_{Z}\right) /\left(S, M_{S}\right)}^{1}
$$

Equivalently, the map

$$
\mathscr{H}^{-1}\left(\mathbb{L}_{i}\right) \rightarrow P^{g p} \otimes \mathcal{O}_{Z}
$$

identifies $\mathbb{L}_{i}$ with $K \otimes \mathcal{O}_{Z}[1]$.

Proof. We may assume that $S$ is affine. To prove the proposition, it suffices to show that the (surjective) map on duals

$$
\operatorname{Hom}\left(P^{g p}, \mathcal{O}_{Z}\right) \rightarrow \operatorname{Hom}_{\mathcal{O}_{Z}}\left(\mathscr{H}^{-1}\left(\mathbb{L}_{i}\right), \mathcal{O}_{Z}\right)
$$

factors through $\operatorname{Hom}\left(K, \mathcal{O}_{Z}\right)$, for then the map

$$
\operatorname{Hom}\left(K, \mathcal{O}_{Z}\right) \rightarrow \operatorname{Hom}_{\mathcal{O}_{Z}}\left(\mathscr{H}^{-1}\left(\mathbb{L}_{i}\right), \mathcal{O}_{Z}\right)
$$

is a surjective morphism of projective $\mathcal{O}_{Z}$-modules of the same rank whence an isomorphism.

To verify that 3.6.2 factors through $\operatorname{Hom}\left(K, \mathcal{O}_{Z}\right)$, it suffices to show that the composite

$$
\operatorname{Hom}\left(P^{g p}, \mathcal{O}_{S}\right) \hookrightarrow \operatorname{Hom}\left(P^{g p}, \mathcal{O}_{Z}\right) \rightarrow \operatorname{Hom}_{\mathcal{O}_{Z}}\left(\mathscr{H}^{-1}\left(\mathbb{L}_{i}\right), \mathcal{O}_{Z}\right)
$$

factors through $\operatorname{Hom}\left(K, \mathcal{O}_{S}\right)$.

For a log scheme $\left(T, M_{T}\right)$ let $\left(T[\epsilon], M_{T[\epsilon]}\right)$ denote the log scheme whose underlying scheme $T[\epsilon]$ is the scheme of dual numbers of $T$ obtained as the spectrum over $T$ of the $\operatorname{ring} \mathcal{O}_{T}[\epsilon] /\left(\epsilon^{2}\right)$. The log structure $M_{T[\epsilon]}$ is induced by the composite map

$$
M_{T} \longrightarrow \mathcal{O}_{T} \stackrel{f \mapsto f}{\longrightarrow} \mathcal{O}_{T}[\epsilon] .
$$


There is a commutative triangle

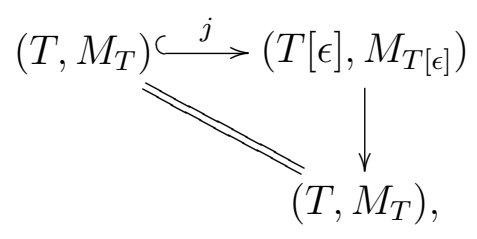

where $j$ is the exact closed immersion defined by setting $\epsilon$ equal to 0 .

This construction defines a lifting

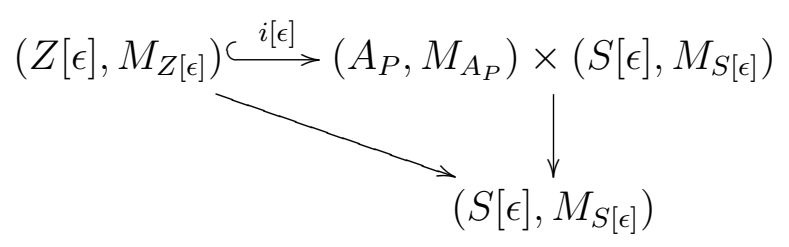

of 1.5.1 to $\mathscr{H}^{\log }\left(\left(S[\epsilon], M_{S[\epsilon]}\right)\right)$. Let $\mathscr{D}_{\epsilon}$ denote the set of commutative diagrams

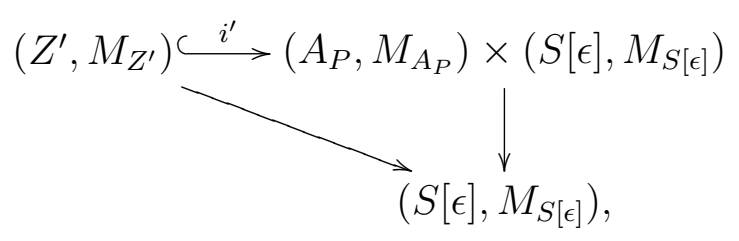

reducing to 1.5.1 modulo $\epsilon$, where $i^{\prime}$ is a (not necessarily $T_{Q}$-equivariant) closed immersion, and $\left(Z^{\prime}, M_{Z^{\prime}}\right) \rightarrow\left(S[\epsilon], M_{S[\epsilon]}\right)$ is log smooth. By [Ol1, 5.2] the lifting 3.6.4 identifies the set $\mathscr{D}_{\epsilon}$ with $\operatorname{Hom}_{\mathcal{O}_{Z}}\left(\mathscr{H}^{-1}\left(\mathbb{L}_{i}\right), \mathcal{O}_{Z}\right)$. On the other hand, we can also view

$$
\operatorname{Hom}\left(P^{g p}, \mathcal{O}_{S}\right) \simeq \operatorname{Ker}\left(T_{P}(S[\epsilon]) \rightarrow T_{P}(S)\right)
$$

as the $S$-valued points of the Lie algebra $\operatorname{Lie}\left(T_{P}\right)$ of $T_{P}$. Unwinding the definitions, one finds that with these identifications the map 3.6.3 sends an element $u \in \operatorname{Lie}\left(T_{P}\right)(S)$ to the element of $\mathscr{D}_{\epsilon}$

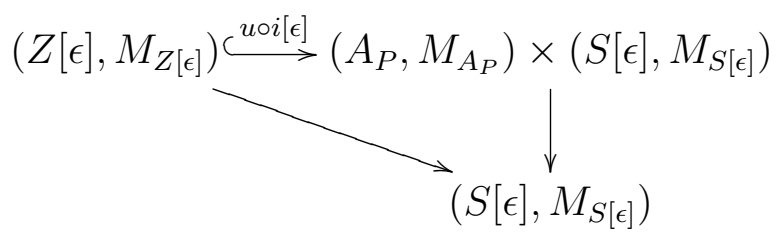

obtained from 3.6.4 by composing the inclusion $i$ with the infinitesimal automorphism $u$ of $\left(A_{P}, M_{A_{P}}\right) \times\left(S[\epsilon], M_{S[\epsilon]}\right)$. Since $i[\epsilon]$ is $T_{Q}$-equivariant, it follows that 3.6 .3 sends $\operatorname{Hom}\left(Q^{g p}, \mathcal{O}_{S}\right)=$ $\operatorname{Lie}\left(T_{Q}\right)(S)$ to zero and therefore factors through $\operatorname{Hom}\left(K, \mathcal{O}_{S}\right)$.

\section{Deformation theory}

4.1. Let $B$ be a ring, $I \subset B$ a square zero ideal, and set $B_{0}:=B / I$. Let $M_{B}$ be a fs $\log$ structure on $\operatorname{Spec}(B)$, and let $M_{B_{0}}$ be the pullback to $\operatorname{Spec}\left(B_{0}\right)$, so we have an exact closed immersion

$$
\left(\operatorname{Spec}\left(B_{0}\right), M_{B_{0}}\right) \hookrightarrow\left(\operatorname{Spec}(B), M_{B}\right)
$$


Let

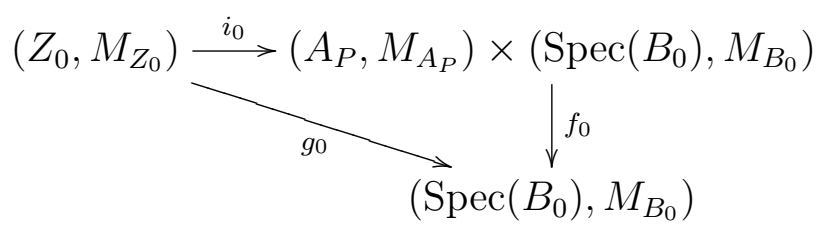

be an element of $\mathscr{H}^{\log }\left(\left(\operatorname{Spec}\left(B_{0}\right), M_{B_{0}}\right)\right)$.

Proposition 4.2. There exists a lifting of 4.1.1 to an element of $\mathscr{H}^{\log }\left(\left(\operatorname{Spec}(B), M_{B}\right)\right)$.

Proof. By [Ol1, 5.6], the obstruction to finding a commutative diagram of fine log schemes (not necessarily $T_{Q}$-invariant)

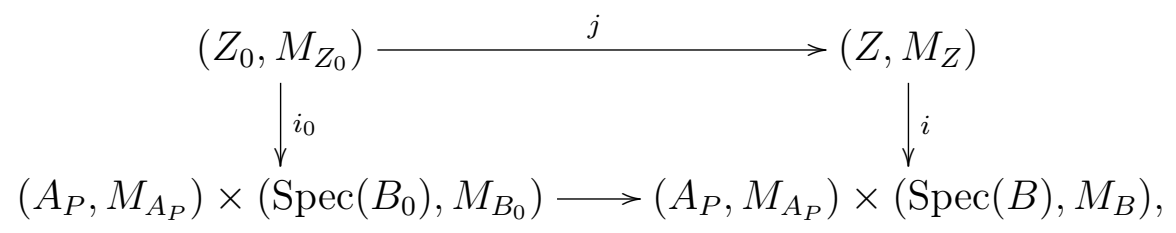

with $\left(Z, M_{Z}\right) \rightarrow\left(\operatorname{Spec}(B), M_{B}\right)$ log smooth, is a class in the group

$$
\operatorname{Ext}^{2}\left(\mathbb{L}_{i_{0}}, I \otimes \mathcal{O}_{Z_{0}}\right) \simeq H^{1}\left(Z_{0}, \mathscr{H}^{-1}\left(\mathbb{L}_{i_{0}}\right)^{*} \otimes I\right)=0
$$

Note that $[\mathrm{Ol1}, 5.6]$ is not stated in sufficient generality, but the proof gives also this result. Therefore we can find a diagram 4.2.1. Let $\mathscr{D}$ denote the set of diagrams 4.2.1. By $[\mathrm{Ol1}, 5.6]$ the set $\mathscr{D}$ admits a natural torsorial action of

$$
\operatorname{Ext}^{1}\left(\mathbb{L}_{i_{0}}, I \otimes \mathcal{O}_{Z_{0}}\right) \simeq H^{0}\left(Z_{0}, \mathscr{H}^{-1}\left(\mathbb{L}_{i_{0}}\right)^{*} \otimes I\right)=\mathscr{H}^{-1}\left(\mathbb{L}_{i_{0}}\right)^{*} \otimes I .
$$

There is an action

$$
T_{Q} \times \mathscr{D} \rightarrow \mathscr{D}, \quad(u, d) \mapsto u * d
$$

of $T_{Q}$ on the set $\mathscr{D}$. A point $u \in T_{Q}(B)$ sends the diagram 4.2.1 to the diagram

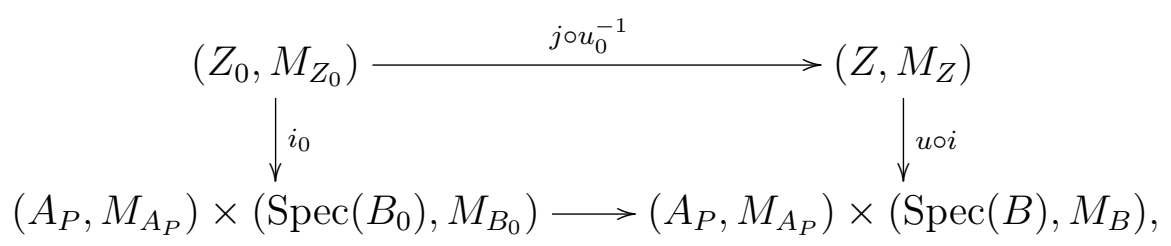

where $u_{0}$ denotes the image of $u$ in $T_{Q}\left(B_{0}\right)$.

Fix an element $d_{0} \in \mathscr{D}$. We then obtain a map

$$
T_{Q}(B) \rightarrow \mathscr{H}^{-1}\left(\mathbb{L}_{i_{0}}\right)^{*} \otimes I, \quad u \mapsto\left[u * d_{0}-d_{0}\right]
$$

This map in fact extends to a morphism of sheaves

$$
T_{Q} \rightarrow \mathscr{H}^{-1}\left(\mathbb{L}_{i_{0}}\right)^{*} \otimes I
$$

on the big étale site of $\operatorname{Spec}(B)$. Indeed to define such a map of sheaves it suffices to specific a distinguished element of $\left(\mathscr{H}^{-1}\left(\mathbb{L}_{i_{0}}\right) \otimes I\right)\left(T_{Q}\right)$. This we obtain by making the base change $T_{Q} \times \operatorname{Spec}(B) \rightarrow \operatorname{Spec}(B)$ and noting that the construction of the map 4.2.2 is compatible with 
smooth base change on $\operatorname{Spec}(B)$. We then obtain the distinguished element of $\left(\mathscr{H}^{-1}\left(\mathbb{L}_{i_{0}}\right) \otimes\right.$ $I)\left(T_{Q}\right)$ by taking the image of the identity map in $T_{Q}\left(T_{Q} \times \operatorname{Spec}(B)\right)$ under the map

$$
T_{Q}\left(T_{Q} \times \operatorname{Spec}(B)\right) \rightarrow \mathscr{H}^{-1}\left(\mathbb{L}_{i_{0}}\right)^{*} \otimes I \otimes \mathcal{O}_{T_{Q}}
$$

obtained from 4.2 .2 by taking $\operatorname{Spec}(B)$ equal to $T_{Q} \times \operatorname{Spec}(B)$ in the above discussion.

It follows from the construction that the map 4.2.3 is compatible with the action of $T_{Q}$ on $\mathscr{H}^{-1}\left(\mathbb{L}_{i_{0}}\right)^{*} \otimes I$ induced by the action on $\mathbb{L}_{i_{0}}$ coming from functoriality of the log cotangent complex in the sense that for any local section $u, v \in T_{Q}$ we have

$$
\rho(u \cdot v)=\rho(v)^{u}+\rho(u) .
$$

By [SGA3, I.5.2.1] this map defines an element in $H^{1}\left(T_{Q}, \mathscr{H}^{-1}\left(\mathbb{L}_{i_{0}}\right)^{*} \otimes I\right)$ (cohomology of the $T_{Q}-\mathcal{O}_{B}$-module in the language of [SGA3, I]). By [SGA3, I.5.3.3] we have $H^{1}\left(T_{Q}, \mathscr{H}^{-1}\left(\mathbb{L}_{i_{0}}\right)^{*} \otimes\right.$ $I)=0$. Therefore there exists an element $e \in \mathscr{H}^{-1}\left(\mathbb{L}_{i_{0}}\right)^{*} \otimes I$ such that $\rho(u)=e^{u}-e$ for all local sections $u \in T_{Q}$. Replacing our choice of lifting $d_{0}$ by $d_{0}-e$ we obtain a $T_{Q}$-invariant lifting.

Corollary 4.3. The set of liftings of 4.1 .1 to $\mathscr{H}^{\log }\left(\left(\operatorname{Spec}(B), M_{B}\right)\right)$ is canonically a torsor under $\operatorname{Hom}(K, I)$.

Proof. The proof of 4.2 also shows that the set of liftings of 4.1 .1 is a torsor under

$$
\operatorname{Hom}\left(\mathscr{H}^{-1}\left(\mathbb{L}_{i_{0}}\right), I \otimes \mathcal{O}_{Z}\right)^{T_{Q}}=\operatorname{Hom}(K, I) .
$$

Corollary 4.4. The diagram

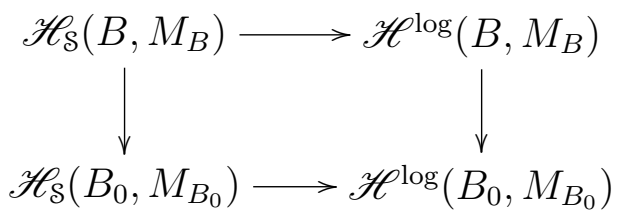

is cartesian.

Remark 4.5. Consider an object 1.5.1 with $S=\operatorname{Spec}(k)$ for some field $k$. Let $J \subset k[P]$ be the ideal defining the closed immersion $Z \hookrightarrow A_{P, k}$. By [H-S, 1.6], the tangent space of the multigraded Hilbert scheme $\mathscr{H}$ at the $k$-valued point $[Z] \in \mathscr{H}(k)$ defined by $Z$ is canonically isomorphic to

$$
\operatorname{Hom}_{\mathscr{O}_{Z}}\left(J / J^{2}, \mathscr{O}_{Z}\right)^{T_{Q}}
$$

On the other hand, the differential $d: J \rightarrow \Omega_{\left(A_{P, k}, M_{A_{P, k}} \times M_{S}\right) /\left(S, M_{S}\right)}^{1} \simeq P^{g p} \otimes_{\mathbb{Z}} k[P]$ induces a map $\bar{d}: J / J^{2} \rightarrow P^{g p} \otimes \mathscr{O}_{Z}$ whose composite with the projection

$$
P^{g p} \otimes \mathscr{O}_{Z} \rightarrow Q^{g p} \otimes \mathscr{O}_{Z} \simeq \Omega_{\left(Z, M_{Z}\right) /\left(S, M_{S}\right)}^{1}
$$

is zero. The map $\bar{d}$ therefore factors through a map $J / J^{2} \rightarrow K \otimes \mathscr{O}_{Z}$, which we again denote by $\bar{d}$. Dualizing we obtain a map

$$
\operatorname{Hom}(K, k) \simeq \operatorname{Hom}_{\mathscr{O}_{Z}}\left(K \otimes \mathscr{O}_{Z}, \mathscr{O}_{Z}\right)^{T_{Q}} \stackrel{\bar{d}^{*}}{\rightarrow} \operatorname{Hom}_{\mathscr{O}_{Z}}\left(J / J^{2}, \mathscr{O}_{Z}\right)^{T_{Q}} .
$$

Unwinding the definitions and granting for the moment 1.7, one finds that this map is identified with the map on tangent spaces induced by the map $\mathcal{S}_{P \rightarrow Q} \hookrightarrow \mathscr{H}$. 


\section{Injectivity OF $\mathscr{H}_{S} \rightarrow \mathscr{H}^{\text {log }}$}

Proposition 5.1. Let $\left(T, M_{T}\right)$ be an fs log scheme. Then the map $\mathscr{H}_{\mathcal{S}}\left(T, M_{T}\right) \rightarrow \mathscr{H}^{\log }\left(T, M_{T}\right)$ is injective.

Proof. Let

$$
f_{1}, f_{2}:\left(T, M_{T}\right) \rightarrow\left(\mathcal{S}, M_{\mathcal{S}}\right)
$$

be two morphisms defining the same element of $\mathscr{H}^{\log }\left(T, M_{T}\right)$. To prove that $f_{1}=f_{2}$, we may by a standard limit argument assume that $T$ is of finite type over $k$. In this case it further suffices to verify that $f_{1}=f_{2}$ at the completion of $T$ at a point, and for this in turn it suffices to verify that $f_{1}=f_{2}$ modulo each power of the maximal ideal. We may therefore assume that $T$ is the spectrum of an artinian local ring. By 4.4 it then suffices to consider the case when $T$ is the spectrum of a field $k^{\prime}$ and there exists an fs monoid $L$ and a morphism $L \rightarrow M_{T}$ inducing an isomorphism $L \simeq \bar{M}_{T}$.

Let $R$ denote the completion of $k^{\prime}[L]$ at the maximal ideal obtained by setting the nonzero elements of $L$ to 0 , let $M_{R}$ denote the $\log$ structure induced by $L \rightarrow R$, and let $\mathfrak{m} \subset R$ be the maximal ideal. Since $\left(\mathcal{S}, M_{\mathcal{S}}\right)$ is $\log$ smooth there exists a morphism

$$
\tilde{f}_{1}:\left(\operatorname{Spec}(R), M_{R}\right) \rightarrow\left(\mathcal{S}, M_{\mathcal{S}}\right)
$$

lifting $f_{1}$. Then by 4.4 there exists a unique morphism

$$
\tilde{f}_{2}:\left(\operatorname{Spec}(R), M_{R}\right) \rightarrow\left(\mathcal{S}, M_{\mathcal{S}}\right)
$$

such that for each integer $n \geq 0$ the elements of $\mathscr{H}^{\log }\left(R_{n}, M_{R_{n}}\right)$ defined by $\tilde{f}_{1}$ and $\tilde{f}_{2}$ are equal, where $R_{n}$ denotes the quotient of $R$ by $\mathfrak{m}^{n+1}$. Since $M_{R}$ is a subsheaf of $\mathcal{O}_{\operatorname{Spec}(R)}$, to verify that $\tilde{f}_{1}=\tilde{f}_{2}$ it suffices to show that the underlying morphisms of $\operatorname{schemes} \operatorname{Spec}(R) \rightarrow \mathcal{S}$ are equal. For this in turn it suffices to show that the composites to the multigraded Hilbert scheme

$$
\operatorname{Spec}(R) \rightarrow \mathcal{S} \rightarrow \mathscr{H}
$$

are equal, since the generic point of $\operatorname{Spec}(R)$ maps to the open subset of $\mathcal{S}$ where $\mathcal{S} \rightarrow \mathscr{H}$ is an open immersion. But the statement that the two morphisms 5.1.1 are equal is clear, because by assumption the morphisms

$$
\operatorname{Spec}\left(R_{n}\right) \rightarrow \mathscr{H}
$$

obtained by reduction modulo each power of $\mathfrak{m}$ are equal.

\section{The FUnCtor $\mathscr{H}^{\log }$ IS Limit PRESERVING}

Proposition 6.1. Let

$$
\cdots \rightarrow\left(\operatorname{Spec}\left(R_{i+1}\right), M_{R_{i+1}}\right) \rightarrow\left(\operatorname{Spec}\left(R_{i}\right), M_{R_{i}}\right) \rightarrow \cdots
$$

be a filtering projective system of noetherian affine $f_{s}$ log schemes with strict transition maps, and set

Then the map

$$
\left(\operatorname{Spec}(R), M_{R}\right):=\lim _{\longleftarrow}\left(\operatorname{Spec}\left(R_{i}\right), M_{R_{i}}\right)
$$

$$
\underset{\lim }{\longrightarrow} \mathscr{H}^{\log }\left(\operatorname{Spec}\left(R_{i}\right), M_{R_{i}}\right) \rightarrow \mathscr{H}^{\log }\left(\operatorname{Spec}(R), M_{R}\right)
$$


is a bijection.

Proof. The injectivity of 6.1 .1 follows from the fact that the classifying stacks of log structures defined in $[\mathrm{Ol} 2]$ are locally of finite type over $\mathbb{Z}$. Moreover, given an object $\left(Z, M_{Z}\right) \hookrightarrow$ $\left(A_{P}, M_{A_{P}}\right) \times\left(\operatorname{Spec}(R), M_{R}\right)$ one can find a $T_{Q}$-equivariant closed immersion

$$
\left(Z_{i}, M_{Z_{i}}\right) \hookrightarrow\left(A_{P}, M_{A_{P}}\right) \times\left(\operatorname{Spec}\left(R_{i}\right), M_{R_{i}}\right)
$$

over some $R_{i}$ inducing $\left(Z, M_{Z}\right)$, such that such that the underlying closed immersion $Z_{i} \hookrightarrow$ $A_{P} \times \operatorname{Spec}\left(R_{i}\right)$ is an element of $\mathscr{H}\left(R_{i}\right)$. The only issue then is to show that after possibly replacing $i$ by some bigger index, the map $\left(Z_{i}, M_{Z_{i}}\right) \rightarrow\left(\operatorname{Spec}\left(R_{i}\right), M_{R_{i}}\right)$ is log smooth. This follows from the following lemma applied to the closed subset $W_{i} \subset Z_{i}$ where $\left(Z_{i}, M_{Z_{i}}\right) \rightarrow$ $\left(\operatorname{Spec}\left(R_{i}\right), M_{R_{i}}\right)$ fails to be $\log$ smooth.

Lemma 6.2. Let $S$ be a scheme, and $Z \hookrightarrow A_{P, S}$ an $S$-valued point of $\mathscr{H}$. Then for any $T_{Q}$-invariant closed subset $W \subset Z$ the image of $W$ in $S$ is closed.

Proof. View $W$ as a scheme with the reduced structure. Let $\pi: Z \rightarrow S$ be the affine projection. The ideal sheaf $J \subset \mathcal{O}_{Z}$ defining $W$ corresponds to a $T_{Q}$-invariant sheaf of ideals $\pi_{*} J \subset \pi_{*} \mathcal{O}_{Z}$ and is therefore given by $\mathcal{O}_{S^{-}}$submodules $\left(\pi_{*} J\right)_{q} \subset\left(\pi_{*} \mathcal{O}_{Z}\right)_{q}$. The image of $W$ in $S$ is then equal to the closed subscheme defined by the ideal $\left(\pi_{*} J\right)_{0} \subset \mathcal{O}_{S}$.

\section{SurJeCtivity of $\mathscr{H}_{S} \rightarrow \mathscr{H}^{\text {log }}$}

The following result completes the proof of 1.7 .

Proposition 7.1. Let $\left(T, M_{T}\right)$ be an fs log scheme. Then the map

$$
\mathscr{H}_{\mathcal{S}}\left(T, M_{T}\right) \rightarrow \mathscr{H}^{\log }\left(T, M_{T}\right)
$$

is surjective.

Proof. Consider first the case when $T=\operatorname{Spec}(k)$ and $M_{T}$ is given by an fs monoid $L$ and a map $L \rightarrow k$ sending all nonzero elements to zero.

Let $\left(Z, M_{Z}\right) \hookrightarrow\left(A_{P}, M_{A_{P}}\right) \times\left(\operatorname{Spec}(k), M_{k}\right)$ be an object of $\mathscr{H}^{\log }\left(\operatorname{Spec}(k), M_{k}\right)$. By assumption the map $P \rightarrow H^{0}\left(Z, M_{\left(Z, M_{Z}\right) /\left(k, M_{k}\right)}\right)$ factors through $Q$.

Lemma 7.2. Let $g:\left(Z, M_{Z}\right) \rightarrow\left(\operatorname{Spec}(k), M_{k}\right)$ be the projection. Then the natural map $k^{*} \oplus L^{g p} \rightarrow H^{0}\left(Z, g^{*} M_{k}^{g p}\right)^{T_{Q}}$ is an isomorphism, where $H^{0}\left(Z, g^{*} M_{k}^{g p}\right)^{T_{Q}} \subset H^{0}\left(Z, g^{*} M_{k}^{g p}\right)$ denotes the sections invariant under $T_{Q}$.

Proof. Note first of all that $g^{-1} \bar{M}_{k}^{g p}$ is the constant sheaf defined by $L^{g p}$, and since $Z$ is connected we therefore have $H^{0}\left(Z, g^{-1} \bar{M}_{k}^{g p}\right)=L^{g p}$. Consideration of the commutative diagram

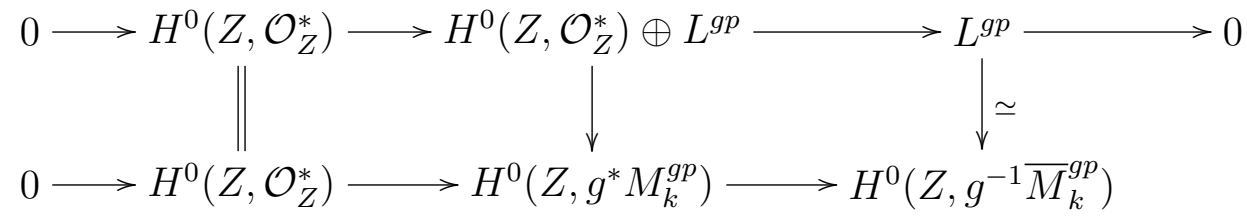

shows that the map

$$
H^{0}\left(Z, \mathcal{O}_{Z}^{*}\right) \oplus L^{g p} \rightarrow H^{0}\left(Z, g^{*} M_{k}^{g p}\right)
$$


is an isomorphism. Since $H^{0}\left(Z, \mathcal{O}_{Z}\right)^{T_{Q}}=k$, this implies the lemma.

Since $K \subset P^{g p}$ maps to zero in $H^{0}\left(Z, M_{\left(Z, M_{Z}\right) /\left(k, M_{k}\right)}^{g p}\right)$ and $T_{Q}$ acts trivially on the image of $K$ in $H^{0}\left(Z, M_{Z}^{g p}\right)$, we obtain a map $\rho: K \rightarrow k^{*} \oplus L^{g p}=H^{0}\left(Z, g^{*} M_{k}^{g p}\right)^{T_{Q}}$. Let $\Sigma \subset K$ denote $\rho^{-1}\left(k^{*} \oplus L\right)$, so that $\Sigma \subset K$ is a submonoid. We then get a map

$$
i^{*}+\rho: P^{g p} \oplus K \rightarrow H^{0}\left(Z, M_{Z}^{g p}\right)
$$

which sends $P \oplus \Sigma$ to $H^{0}\left(Z, M_{Z}\right)$. Let $E_{\Sigma} \subset Q \oplus P^{g p}$ denote the image of $P \oplus \Sigma$ as in 2.4. Since the composite

$$
K \longrightarrow P^{g p} \oplus K \longrightarrow H^{0}\left(Z, M_{Z}^{g p}\right)
$$

is by construction zero, where the first arrow is $k \mapsto(k,-k)$, the map 7.2.1 descends to $E_{\Sigma}$ and we obtain a commutative diagram

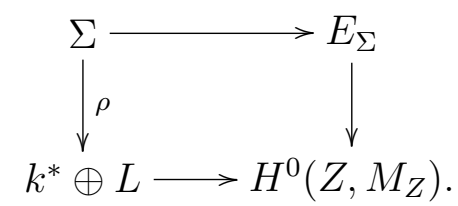

Let $\left(V_{\Sigma}, M_{V_{\Sigma}}\right) \subset\left(A_{P}, M_{A_{P}}\right) \times\left(\operatorname{Spec}(k), M_{k}\right)$ denote the closed immersion obtained from the fiber product

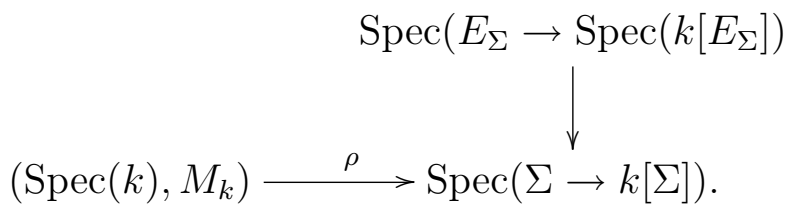

The diagram 7.2.2 then induces a morphism of log schemes compatible with the $T_{Q}$-actions

$$
r:\left(Z, M_{Z}\right) \rightarrow\left(V_{\Sigma}, M_{\Sigma}\right)
$$

which is a closed immersion since both are closed in $\left(A_{P}, M_{A_{P}}\right) \times\left(\operatorname{Spec}(k), M_{k}\right)$. We claim that $r$ is an isomorphism.

Lemma 7.3. The morphism $r$ is log étale.

Proof. Both $\left(Z, M_{Z}\right)$ and $\left(V_{\Sigma}, M_{V_{\Sigma}}\right)$ are $\log$ smooth over $\left(\operatorname{Spec}(k), M_{k}\right)$. It therefore suffices to show that the pullback map

$$
r^{*} \Omega_{\left(V_{\Sigma}, M_{V_{\Sigma}}\right) /\left(\operatorname{Spec}(k) M_{k}\right)}^{1} \rightarrow \Omega_{\left(Z, M_{Z}\right) /\left(\operatorname{Spec}(k), M_{k}\right)}^{1}
$$

is an isomorphism. This is clear because this map is canonically identified with the identity map

$$
\mathcal{O}_{Z} \otimes Q^{g p} \rightarrow \mathcal{O}_{Z} \otimes Q^{g p}
$$

Lemma 7.4. Let $f:\left(X, M_{X}\right) \rightarrow\left(Y, M_{Y}\right)$ be a log étale closed immersion, and let $\left(X^{\mathrm{sat}}, M_{X^{\mathrm{sat}}}\right)$ (resp. $\left.\left(Y^{\mathrm{sat}}, M_{Y^{\mathrm{sat}}}\right)\right)$ be the saturation of $\left(X, M_{X}\right)$ (resp. $\left.\left(Y, M_{Y}\right)\right)$. Then the map

$$
f^{\text {sat }}:\left(X^{\text {sat }}, M_{X^{\text {sat }}}\right) \rightarrow\left(Y^{\text {sat }}, M_{Y^{\text {sat }}}\right)
$$

is a strict closed and open immersion. 
Proof. Let $\bar{x} \rightarrow X$ be a geometric point, and choose a a finitely generated group $G$ and a map $G \rightarrow M_{Y, \bar{x}}^{g p}$ such that the induced map $G \rightarrow \bar{M}_{Y, \bar{x}}^{g p}$ is surjective. Since $f$ is a closed immersion the composite map

$$
G \rightarrow \bar{M}_{Y, \bar{x}}^{g p} \rightarrow \bar{M}_{X, \bar{x}}^{g p}
$$

is also surjective. Define

$$
P_{Y}:=G \times_{M_{Y, \bar{x}}^{g p}} M_{Y, \bar{x}}, \quad P_{X}:=G \times_{M_{X, \bar{x}}^{g p}} M_{X, \bar{x}}
$$

so we have an inclusion $i: P_{Y} \subset P_{X}$. As in [Og1, 2.2.11], after replacing $Y$ by an étale neighborhood of $\bar{x}$, the maps $P_{Y} \rightarrow M_{Y, \bar{x}}$ and $P_{X} \rightarrow M_{X, \bar{x}}$ extend to charts so we have a commutative diagram

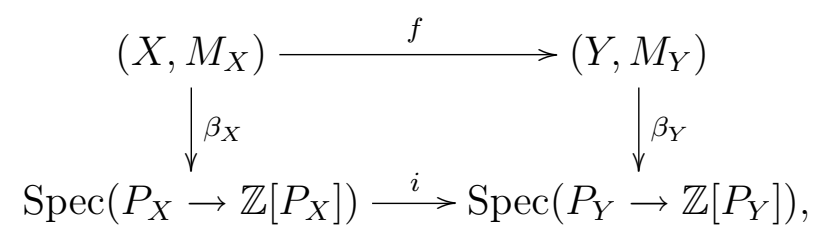

where the maps $\beta_{X}$ and $\beta_{Y}$ are strict. Now observe that since $\bar{M}_{Y, \bar{x}}^{g p} \rightarrow \bar{M}_{X, \bar{x}}^{g p}$ is surjective with kernel a finite group (since $f$ is log étale), the map $P_{Y} \rightarrow P_{X}$ induces an isomorphism on saturations. Since

$$
X^{\text {sat }}=\operatorname{Spec}\left(\mathbb{Z}\left[P_{X}^{\mathrm{sat}}\right]\right) \times_{\operatorname{Spec}\left(\mathbb{Z}\left[P_{X}\right]\right)} X \quad\left(\operatorname{resp} . Y^{\text {sat }}=\operatorname{Spec}\left(\mathbb{Z}\left[P_{Y}^{\mathrm{sat}}\right]\right) \times_{\operatorname{Spec}\left(\mathbb{Z}\left[P_{Y}\right]\right)} Y\right)
$$

with $M_{X^{\text {sat }}}\left(\right.$ resp. $\left.M_{Y^{\text {sat }}}\right)$ induced by the natural map $P_{X}^{\text {sat }} \rightarrow \mathcal{O}_{X^{\text {sat }}}\left(\operatorname{resp} . P_{Y}^{\text {sat }} \rightarrow \mathcal{O}_{Y^{\text {sat }}}\right.$ ), this implies that $f^{\text {sat }}$ is a strict closed immersion.

On the other hand, from the commutative diagram

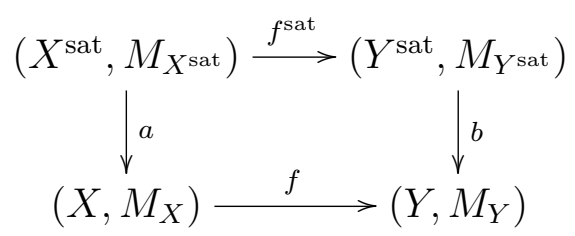

where $a, b$, and $f$ are log étale, we deduce that $f^{\text {sat }}$ is also log étale, and hence since $f^{\text {sat }}$ is strict the map $X^{\text {sat }} \rightarrow Y^{\text {sat }}$ is étale in the usual sense. It follows that $f^{\text {sat }}$ is also an open immersion.

Lemma 7.5. Let $\left(\operatorname{Spec}(k), M_{k}\right)$ be as in the beginning of the proof of 7.1 (so we have a chart $L \rightarrow M_{k}$ ), and let $L \rightarrow N$ be a morphism of fine monoids with $N^{\text {gp }}$ torsion free. Set $\left(W, M_{W}\right):=\operatorname{Spec}\left(N \rightarrow k \otimes_{k[L]} k[N]\right)$. Then any log étale closed immersion $\left(Z, M_{Z}\right) \hookrightarrow$ $\left(W, M_{W}\right)$ is an open and closed immersion.

Proof. Let $\left(\mathcal{W}, M_{\mathcal{W}}\right)$ denote $\operatorname{Spec}(N \rightarrow k[N])$, so that there is a strict closed immersion $\left(W, M_{W}\right) \hookrightarrow\left(\mathcal{W}, M_{\mathcal{W}}\right)$. Let $z \in Z$ be a $k$-valued closed point and let $\widehat{\mathcal{O}}_{\mathcal{W}, z}$ denote the completion of the local ring of $\mathcal{W}$ at $z$. We have $\left(\mathcal{W}^{\text {sat }}, M_{\mathcal{W}_{\text {sat }}}\right)$ equal to $\operatorname{Spec}\left(N^{\text {sat }} \rightarrow k\left[N^{\text {sat }}\right]\right)$. Let $\widehat{\mathcal{O}}_{\mathcal{W}^{\text {sat }, z}}$ denote the coordinate ring of the scheme $\operatorname{Spec}\left(\widehat{\mathcal{O}}_{\mathcal{W}, z}\right) \times_{\mathcal{W}} \mathcal{W}^{\text {sat }}$, so that $\widehat{\mathcal{O}}_{\mathcal{W}^{\text {sat }}, z}$ is a finite $\widehat{\mathcal{O}}_{\mathcal{W}, z}$-algebra. Let $\left(Z_{z}, M_{Z_{z}}\right)$ denote the fiber product $\left(Z, M_{Z}\right) \times_{\left(\mathcal{W}, M_{\mathcal{W}}\right)}\left(\operatorname{Spec}\left(\widehat{\mathcal{O}}_{\mathcal{W}, z}\right), M_{\widehat{\mathcal{O}}_{\mathcal{W}, z}}\right)$. 
Since $\left(Z, M_{Z}\right) \hookrightarrow\left(W, M_{W}\right)$ is log étale, $\left(Z_{z}, M_{Z_{z}}\right)$ lifts uniquely to a log étale closed immer$\operatorname{sion}\left(\mathcal{Z}_{z}, M_{\mathcal{Z}_{z}}\right) \hookrightarrow\left(\operatorname{Spec}\left(\widehat{\mathcal{O}}_{\mathcal{W}, z}\right), M_{\widehat{\mathcal{O}}_{\mathcal{W}, z}}\right)$. On the other hand, since $N^{g p}$ is torsion free, both $\widehat{\mathcal{O}}_{\mathcal{W}, z}$ and $\widehat{\mathcal{O}}_{\mathcal{W}}^{\text {sat }, z}$ are integral domains. By 7.4 we conclude that the map

$$
\left(\mathcal{Z}_{z}^{\text {sat }}, M_{\mathcal{Z}_{z}^{\text {sat }}}\right) \rightarrow\left(\operatorname{Spec}\left(\widehat{\mathcal{O}}_{\mathcal{W}^{\text {sat }}, z}\right), M_{\widehat{\mathcal{O}}_{\mathcal{W}_{\text {sat }, z}}}\right)
$$

is an isomorphism. Since $\widehat{\mathcal{O}}_{\mathcal{W}, z} \rightarrow \widehat{\mathcal{O}}_{\mathcal{W}}{ }_{\text {sat }, z}$ is injective, it follows that $\mathcal{Z}_{z} \rightarrow \operatorname{Spec}\left(\widehat{\mathcal{O}}_{\mathcal{W}, z}\right)$ is étale at $z$. We conclude that $Z \rightarrow W$ is an open and closed immersion.

To check that the map $M_{W} \rightarrow M_{Z}$ is an isomorphism, we show that the map $\bar{M}_{\widehat{\mathcal{O}}_{\mathcal{W}, z}} \rightarrow$ $\bar{M}_{Z, z}$ is an isomorphism. Let $S$ denote the localization of $N$ at the face of elements mapping to units in $k(z)$, and let $\beta: S \rightarrow k(z)$ be the map defining $z$. Then $\widehat{\mathcal{O}}_{\mathcal{W}, z}$ is equal to the completion $k[[S]]$ of $k[S]$ along the ideal defined by the elements $\beta(s)-e_{s}$, and $\bar{M}_{\widehat{\mathcal{W}}, z}$ is equal to $S / S^{*}$. Let $s_{1}, s_{2} \in S$ be two elements whose images in $\bar{M}_{Z, z}$ are equal. Then there exists an element $w \in k[[S]]^{*}$ such that $e_{s_{1}}=w \cdot e_{s_{2}}$. If $\bar{S}$ denotes $S / S^{*}$ then the torus $D\left(\bar{S}^{g p}\right)$ acts on the reduction $k[[S]] / \mathfrak{m}^{n+1}$ of $k[[S]]$ modulo each power of the maximal ideal. Now observe that since $\bar{S}$ is sharp, if

$$
k[[S]] / \mathfrak{m}^{n+1}=\bigoplus_{\bar{s} \in \bar{S}_{\bar{s}}} T_{\bar{s}}^{(n+1)}
$$

denotes the character decomposition of $k[[S]] / \mathfrak{m}^{n+1}$ with respect to the $D\left(\bar{S}^{g p}\right)$-action, then each element of $T_{\bar{s}}^{(n+1)}$ for $\bar{s} \neq 0$ is in the maximal ideal. On the other hand any point $u \in D\left(\bar{S}^{g p}\right)(k)$ sends $w$ to $u\left(s_{1}-s_{2}\right) \cdot w$. Consequently, $s_{1}-s_{2} \in S^{*}$ which implies that $s_{1}$ and $s_{2}$ have the same image in $\bar{S}$.

Applying 7.5 to 7.2 .3 , we conclude that our morphism $r$ is an isomorphism (since $V_{\Sigma}$ is connected). By A.1 this also implies that the map $\Sigma \rightarrow E_{\Sigma}$ is integral.

This completes the proof of 7.1 in the case when $T$ is the spectrum of a field. From 4.4 we then deduce that 7.1 also holds when $T$ is the spectrum of an artinian local ring.

Next we consider the case when $T=\operatorname{Spec}(R)$ is the spectrum of a complete noetherian local ring $R$. Let $\mathfrak{m} \subset R$ be the maximal ideal, and for any $n \geq 0$ let $R_{n}$ denote the quotient $R / \mathfrak{m}^{n+1}$. Let $\left(Z, M_{Z}\right) \hookrightarrow\left(A_{P}, M_{A_{P}}\right) \times\left(T, M_{T}\right)$ be an element of $\mathscr{H}^{\log }\left(T, M_{T}\right)$. For every $n \geq 0$, the reduction of this object modulo $\mathfrak{m}^{n+1}$ defines a unique morphism of fine $\log$ schemes $f_{n}$ : $\left(\operatorname{Spec}\left(R_{n}\right), M_{R_{n}}\right) \rightarrow\left(\mathcal{S}_{P \rightarrow Q}, M_{\mathcal{S}_{P \rightarrow Q}}\right)$, by the case of an artinian local ring already considered. It follows that we obtain a morphism $f:\left(\operatorname{Spec}(R), M_{R}\right) \rightarrow\left(\mathcal{S}_{P \rightarrow Q}, M_{\mathcal{S}_{P \rightarrow Q}}\right)$ inducing the reduction of $\left(Z, M_{Z}\right)$ modulo each power of the maximal ideal. In particular, the pullback of the scheme $Z \rightarrow \mathcal{S}_{P \rightarrow Q}$ along $f$ is equal to $Z$. On $Z$ we therefore obtain a second quotient $i^{*} M_{A_{P} \times T} \rightarrow M_{Z}^{\prime}$ such that modulo each power of the maximal ideal this quotient is equal to $M_{Z}$. We wish to show that $M_{Z}^{\prime}$ is equal to $M_{Z}$. For a geometric point $\bar{z} \rightarrow Z$ with image in the closed fiber, this follows from looking first at the completion of $Z$ at $z$ and then at the reductions modulo the maximal ideal. By specialization it follows that the two quotients are equal at any point of $z$ whose closure meets the closed fiber of $Z$. Finally note that both quotients are $T_{Q}$-equivariant so to verify that the two quotients agree at a point $z \in Z$ it suffices to verify that they agree at some other point in the $T_{Q}$-orbit of $z$. By 6.2 any point $z \in Z$ contains a point $z^{\prime} \in Z$ in its orbit such that the closure of $z^{\prime}$ meets the closed fiber of $Z$. This therefore completes the proof of 7.1 in the case of a complete noetherian local ring. 
We now finish the proof of 7.1 by applying the Artin approximation theorem. So let $\left(T, M_{T}\right)$ be any fs log scheme and $\left(Z, M_{Z}\right) \hookrightarrow\left(A_{P}, M_{A_{P}}\right) \times\left(T, M_{T}\right)$ an element of $\mathscr{H}^{\log }\left(T, M_{T}\right)$. By the injectivity of $\mathscr{H}_{S} \rightarrow \mathscr{H}^{\text {log }}$, to prove that this element of $\mathscr{H}^{\log }\left(T, M_{T}\right)$ is in the image of $\mathscr{H}_{\mathcal{S}}\left(T, M_{T}\right)$ we may work étale locally on $T$. We may therefore assume that $T$ is an affine scheme, and by 6.1 of finite type over $k$. Define

$$
F:(T \text {-schemes })^{\text {op }} \rightarrow \text { Set }
$$

to be the functor which to any $v: T^{\prime} \rightarrow T$ associates the set of morphisms $f:\left(T^{\prime}, v^{*} M_{T}\right) \rightarrow$ $\left(\mathcal{S}_{P \rightarrow Q}, M_{\mathcal{S}_{P \rightarrow Q}}\right)$ such that the image of $f$ under the map

$$
\mathscr{H}_{\mathcal{S}}\left(T^{\prime}, v^{*} M_{T}\right) \rightarrow \mathscr{H}^{\log }\left(T^{\prime}, v^{*} M_{T}\right)
$$

is equal to the restriction to $\left(T^{\prime}, v^{*} M_{T}\right)$ of our given object $\left(Z, M_{Z}\right) \hookrightarrow\left(A_{P}, M_{A_{P}}\right) \times\left(T, M_{T}\right)$. Using 6.1 one sees that $F$ is a limit preserving functor. By the case of a complete noetherian local ring and the Artin approximation theorem [A, 1.12], we conclude that there exists an étale covering $T^{\prime} \rightarrow T$ such that $F\left(T^{\prime}\right)$ is nonempty. This gives the desired morphism to $\left(\mathcal{S}_{P \rightarrow Q}, M_{\mathcal{S}_{P \rightarrow Q}}\right)$ and therefore completes the proof of 7.1 .

\section{Representability of $\mathscr{H}_{\mathbb{Z}}^{\log }$}

8.1. Let $\left(\mathcal{S}_{P \rightarrow Q, \mathbb{Z}}, M_{\mathcal{S}_{P \rightarrow Q, \mathbb{Z}}}\right)$ denote the fs log scheme over $\operatorname{Spec}(\mathbb{Z})$ defined by the fan in 2.5 . The constructions in 2.2 and 2.7 define an object of $\mathscr{H}_{\mathbb{Z}}^{\log }\left(\left(\mathcal{S}_{P \rightarrow Q, \mathbb{Z}}, M_{\mathcal{S}_{P \rightarrow Q, \mathbb{Z}}}\right)\right)$, and hence a morphism of functors

$$
\mathscr{H}_{\mathcal{S}, \mathbb{Z}} \rightarrow \mathscr{H}_{\mathbb{Z}}^{\log }
$$

where $\mathscr{H}_{\mathcal{S}, \mathbb{Z}}$ denotes the functor on the category of fs log schemes represented by $\left(\mathcal{S}_{P \rightarrow Q, \mathbb{Z}}, M_{\mathcal{S}_{P \rightarrow Q, \mathbb{Z}}}\right)$.

Proposition 8.2. The map $\mathcal{S}_{P \rightarrow Q, \mathbb{Z}} \rightarrow \mathscr{H}$ from $\mathcal{S}_{P \rightarrow Q, \mathbb{Z}}$ to the multigraded Hilbert scheme (over $\mathbb{Z}$ ) defined by the family over $\mathcal{S}_{P \rightarrow Q, \mathbb{Z}}$ is proper.

Proof. We verify the valuative criterion for properness. Let $V$ be a complete discrete valuation ring with algebraically closed residue field $k$ and fraction field $F$. Suppose given a commutative diagram of solid arrows

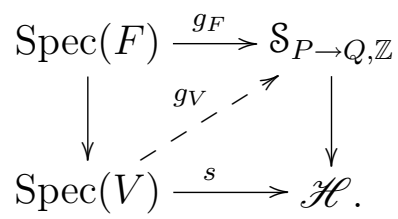

We need to show that there exists a dotted arrow $g_{V}$ filling in the diagram. Moreover, it suffices to consider the case when $g_{F}$ factors through the dense open subset

$$
\operatorname{Spec}(\mathbb{Z}[K]) \subset \mathcal{S}_{P \rightarrow Q, \mathbb{Z}}
$$


Let $\rho: K \rightarrow F^{*}$ be the map defining $g_{F}$, and let $V[P] \longrightarrow R$ be the $T_{Q^{-} \text {-equivariant }}$ surjection defining $s$. We then have a commutative diagram

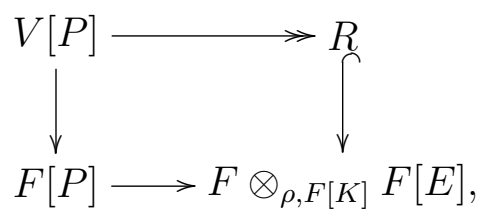

where $E \subset Q \oplus P^{g p}$ is defined as in 2.2. Let $L \subset K$ be the submonoid of elements $n \in K$ with $\rho(n) \in V$, and let $\rho_{V}: L \rightarrow V$ be the map induced by $\rho$. Then the map $P \oplus L \rightarrow R$ induced by the surjection $V \otimes_{\rho_{V}, V[L]} V[P \oplus L] \simeq V[P] \rightarrow R$ factors through $E_{L} \subset L$ since for this it suffices to show that the composite

$$
P \oplus L \rightarrow R \rightarrow F \otimes_{\rho, F[K]} F[E]
$$

factors through $E$ which is clear. We therefore get a commutative diagram

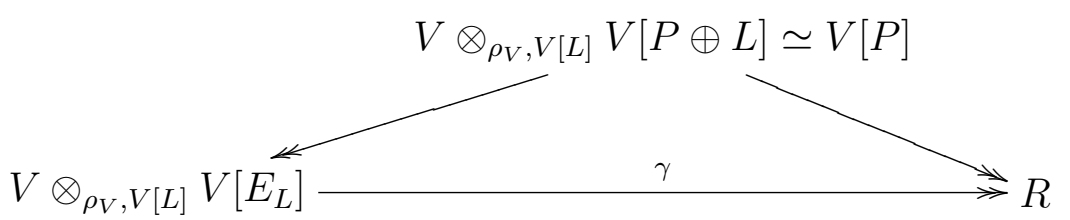

Lemma 8.3. The map $L \rightarrow E_{L}$ is integral.

Proof. The cokernel of the map $L \rightarrow E_{L}$ is equal to $Q$. Let $\pi: E_{L} \rightarrow Q$ be the projection. It suffices to show that for any $q \in Q$ there exists a lifting $\tilde{q}_{0} \in \pi^{-1}(q)$ such that for any element $\tilde{q} \in \pi^{-1}(q)$ there exists an element $l \in L$ such that $\tilde{q}=\tilde{q}_{0}+l$.

For this let $R_{q} \subset R$ be the $q$-eigenspace of $R$ with respect to the $T_{Q^{-}}$-action. By assumption, $R_{q}$ is a free $V$-module of rank 1 . Since the map $V \otimes_{\rho_{V}, V[L]} V\left[E_{L}\right] \rightarrow R$ is surjective, there exists an element $\tilde{q}_{0} \in \pi^{-1}(q)$ such that $\tilde{q}_{0}$ maps to a basis for $R_{q}$. If $\tilde{q} \in \pi^{-1}(q)$ is any other element, then $\tilde{q}-\tilde{q}_{0}$ is an element of $K$ and $\rho\left(\tilde{q}-\tilde{q}_{0}\right)$ is in $V$ since $\tilde{q}$ maps to a multiple of the image of $\tilde{q}_{0}$ in $R_{q}$. It follows that $\tilde{q}-\tilde{q}_{0} \in L$.

It follows that $V \rightarrow V \otimes_{\rho_{V}, V[L]} V\left[E_{L}\right]$ is flat, and therefore the map $\gamma$ is an isomorphism, since for every $q$ the map on eigenspaces

$$
\gamma_{q}:\left(V \otimes_{\rho_{V}, V[L]} V\left[E_{L}\right]\right)_{q} \rightarrow R_{q}
$$

is a surjective map of rank 1 free $V$-modules, and therefore an isomorphism. From this we see that $s$ is obtained by pullback along the map $\rho_{V}: \mathbb{Z}[L] \rightarrow V$ from the family over $\operatorname{Spec}(\mathbb{Z}[L]) \subset \mathcal{S}_{P \rightarrow Q, \mathbb{Z}}$

Corollary 8.4. The morphism $\mathcal{S}_{P \rightarrow Q, \mathbb{Z}} \rightarrow \mathscr{H}$ identifies $\mathcal{S}_{P \rightarrow Q, \mathbb{Z}}$ with the normalization of the closure of the image of the map

$$
T_{Q} \rightarrow \mathscr{H}, \quad u \mapsto u * p_{0}
$$

where $p_{0} \in \mathscr{H}(\mathbb{Z})$ is the point defined by $j: A_{Q} \hookrightarrow A_{P}$.

Proof. The projection $\mathcal{S}_{P \rightarrow Q, \mathbb{Z}} \rightarrow \mathscr{H}$ is quasi-finite, as this can be verified after base change to a field valued point of $\operatorname{Spec}(\mathbb{Z})$, where we already saw the result. Since the morphism is also proper, it is finite. Since $\mathcal{S}_{P \rightarrow Q, \mathbb{Z}}$ is normal, it follows that $\mathcal{S}_{P \rightarrow Q, \mathbb{Z}}$ is equal to the normalization 
of the closure in $\mathscr{H}$ of the image of the map $S_{P \rightarrow Q, \mathbb{Z}} \times \operatorname{Spec}(\mathbb{Q}) \rightarrow \mathscr{H} \times \operatorname{Spec}(\mathbb{Q})$, where we again know the result by the case of a field.

Theorem 8.5. The map 8.1.1 is an isomorphism.

Proof. Note first of all that the results of sections 3, 4, and 6 hold also over an arbitrary base scheme by exactly the same arguments.

To see that for any fs log scheme $\left(T, M_{T}\right)$ the map

$$
\mathscr{H}_{\mathrm{S}, \mathbb{Z}}\left(T, M_{T}\right) \rightarrow \mathscr{H}_{\mathbb{Z}}^{\log }\left(T, M_{T}\right)
$$

is injective, note that by the same argument used at the beginning of the proof of 5.1 it suffices to consider the case when $T$ is the spectrum of a field, and in this case we have already shown the result.

The surjectivity of $\mathscr{H}_{\S, \mathbb{Z}} \rightarrow \mathscr{H}_{\mathbb{Z}}^{\text {log }}$ is also reduced to the case of a field by the same argument used in the proof of 7.1 .

\section{APPEndix A. A CRITERION FOR INTEGRALITY}

Proposition A.1. Let $L$ be a fine monoid and assume that $\left(L / L^{*}\right)^{g p}$ and $L^{g p}$ are torsion free. Let $L \rightarrow P$ an injective morphism of fine monoids. Let $k$ be a field and assume that the cokernel of the map $L^{g p} \rightarrow P^{g p}$ has order invertible in $k$. Let $\beta: L \rightarrow k$ be a morphism of monoids such that $\beta^{-1}\left(k^{*}\right)=L^{*}$. Then $L \rightarrow P$ is integral if and only if the morphism of log schemes

$$
\operatorname{Spec}\left(P \rightarrow k \otimes_{\beta, k[L]} k[P]\right) \rightarrow \operatorname{Spec}(L \stackrel{\beta}{\rightarrow} k)
$$

is integral.

Proof. We may without loss of generality assume that $k$ is algebraically closed.

Let $\beta_{0}: L \rightarrow k$ be the map obtained by composing the projection $L \rightarrow L / L^{*}$ with the map $L / L^{*} \rightarrow k$ sending all nonzero elements to 0 . Let $\gamma: L^{*} \rightarrow k^{*}$ be the map induced by $\beta$. Since $\left(L / L^{*}\right)^{g p}$ is torsion free, there exists a map $\tilde{\gamma}: L^{g p} \rightarrow k^{*}$ extending $\gamma$. This map $\tilde{\gamma}$ defines an isomorphism of log schemes

$$
\tilde{\gamma},: \operatorname{Spec}(L \stackrel{\beta}{\longrightarrow} k) \rightarrow \operatorname{Spec}\left(L \stackrel{\beta_{0}}{\longrightarrow} k\right)
$$

which is the identity on $\operatorname{Spec}(k)$ and for which the map on log structures is induced by the map

$$
L \rightarrow k^{*} \oplus L, \quad \ell \mapsto\left(\tilde{\gamma}(\ell)^{-1}, \ell\right)
$$

Since $k$ is algebraically closed and $L^{g p} \rightarrow P^{g p}$ is injective, we can also choose a map $\epsilon: P^{g p} \rightarrow$ $k^{*}$ extending $\tilde{\gamma}$. We then obtain a commutative diagram

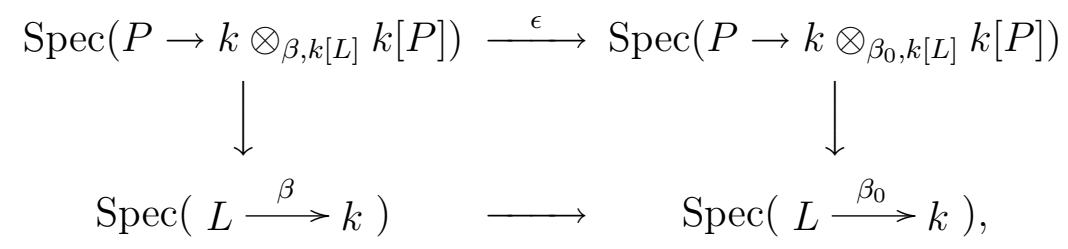


where the top horizontal arrow is the isomorphism induced by the map

$$
P \rightarrow k^{*} \oplus P, \quad p \mapsto\left(\epsilon^{-1}(p), p\right) .
$$

It follows that it suffices to consider the case when $\beta=\beta_{0}$, which we assume for the rest of the proof.

Let $z_{0} \in \operatorname{Spec}(k[L])$ denote the point defined by $\beta$.

The "only if" direction of the proposition is immediate.

For the "if" direction, note that it suffices to show that the morphism of rings $k[L] \rightarrow k[P]$ is flat. Let $G$ denote $P^{g p} / L^{g p}$ so we have an exact sequence of diagonalizable group schemes

$$
0 \rightarrow D(G) \rightarrow D\left(P^{g p}\right) \rightarrow D\left(L^{g p}\right) \rightarrow 0 .
$$

The group $D\left(P^{g p}\right)$ acts on $\operatorname{Spec}(k[P])$ covering the action of $D\left(L^{g p}\right)$ on $\operatorname{Spec}(k[L])$. In particular, restricting the $D\left(P^{g p}\right)$-action to $D(G)$ we obtain an action of $D(G)$ on $k[P]$ over $k[L]$. Let $k[P]=\oplus_{g \in G} M_{g}$ be the character decomposition of $k[P]$ with respect to this action. We need to show that each of the $k[L]$-modules $M_{g}$ is flat over $k[L]$. Note that because $D\left(P^{g p}\right)$ is commutative, the action of $D\left(P^{g p}\right)$ induces an action of $D\left(P^{g p}\right)$ on each $M_{g}$ covering the action of $D\left(P^{g p}\right)$ on $k[L]$ induced by the projection $D\left(P^{g p}\right) \rightarrow D\left(L^{g p}\right)$. It follows that the closed set $Z_{g} \subset \operatorname{Spec}(k[L])$ of points $z \in \operatorname{Spec}(k[L])$ where the stalk of $M_{g}$ is not flat over $\mathcal{O}_{\operatorname{Spec}(k[L]), z}$ is $D\left(L^{g p}\right)$ invariant.

Lemma A.2. Any nonempty $D\left(L^{g p}\right)$-invariant closed subset $Z \subset \operatorname{Spec}(k[L])$ contains $z_{0}$.

Proof. Let $z \in Z(k)$ be a closed point defined by a morphism $\gamma: L \rightarrow k$. Since $L / L^{*}$ is $\operatorname{sharp}$ and $\left(L / L^{*}\right)^{g p}$ is torsion free, there exists a morphism of monoids $h: L / L^{*} \rightarrow \mathbb{N}$ such that $h^{-1}(0)=0$. Consider the morphism

$$
\rho: \mathbb{A}_{k}^{1} \times D\left(L^{g p}\right) \rightarrow \operatorname{Spec}(k[L])
$$

defined by the map $L \rightarrow k\left[t, L^{g p}\right]$ sending $l$ to $\gamma(l) t^{h(l)} e_{l}$. Then $\rho(1,1)=z$ and if $\tilde{\gamma} \in D\left(L^{g p}\right)$ is an element extending $\left.\gamma\right|_{L^{*}}$, then $\rho\left(0, \tilde{\gamma}^{-1}\right)=z_{0}$. Since the restriction of $\rho$ to $\mathbb{G}_{m} \times D\left(L^{g p}\right)$ is equal to the composite

$$
\mathbb{G}_{m} \times D\left(L^{g p}\right) \stackrel{h+\mathrm{id}}{\longrightarrow} D\left(L^{g p}\right) \stackrel{u \mapsto u * z}{\longrightarrow} Z
$$

and $Z$ is closed, it follows that the image of $\rho$ is contained in $Z$.

It follows that to prove the integrality of the map $L \rightarrow P$, it suffices to show that the stalks of the $M_{g}$ at the point $z_{0} \in \operatorname{Spec}(k[L])$ are flat, and for this in turn it suffices to prove that the reductions modulo each power of the maximal ideal $\mathfrak{m}_{z_{0}}$ of $k[L]$ are flat. By assumption the morphism A.1.1 is integral. It follows that for each $n \geq 0$ the morphism of $\log$ schemes

$$
\operatorname{Spec}\left(P \rightarrow\left(k[L] / \mathfrak{m}_{z_{0}}^{n+1}\right) \otimes_{k[L]} k[P]\right) \rightarrow \operatorname{Spec}\left(L \rightarrow k[L] / \mathfrak{m}_{z_{0}}^{n+1}\right)
$$

is $\log$ smooth and integral. Since a log smooth and integral morphism is flat, this concludes the proof that $L \rightarrow P$ is integral. 


\section{REFERENCES}

[A] M. Artin, Algebraic approximation of structures over complete local rings, Inst. Hautes tudes Sci. Publ. Math. 36 (1969), 23-58.

[C] O. Chuvashova, The main component of the toric Hilbert scheme, preprint (2007).

[SGA3] M. Demazure and A. Grothendieck, Schémas en Groupes, Lecture Notes in Math. 151, 152, 153, Springer-Verlag, Berlin (1970).

[H-S] M. Haiman and B. Sturmfels, Multigraded Hilbert schemes, J. Algebraic Geom. 13 (2004), 725-769.

[K] K. Kato, Logarithmic structures of Fontaine and Illusie, in Algebraic analysis, geometry and number theory: Proceedings of the JAMI Inaugural Conference, 191-224, Johns Hopkins Univ. Press, Baltimore, MD, 1989

[Og1] A. Ogus, Lectures on logarithmic algebraic geometry, preprint (2007).

[Og2] _ F-crystals, Griffiths transversality, and the Hodge decomposition, Astérisque 221 (1994).

[Ol1] M. Olsson, The logarithmic cotangent complex, Math. Ann. 333 (2005), 859-931.

[Ol2] _ L L Logarithmic geometry and algebraic stacks, Ann. Sci. d'ENS 36 (2003), 747-791. 